\title{
Acclimatation en France de Ptilodactyla exotica espèce à mœurs anthropophiles (Coleoptera, Ptilodactylidae)
}

Henri-Pierre Aberlenc, Roland Allemand

\section{Citer ce document / Cite this document :}

Aberlenc Henri-Pierre, Allemand Roland. Acclimatation en France de Ptilodactyla exotica espèce à mœurs anthropophiles (Coleoptera, Ptilodactylidae). In: Bulletin de la Société entomologique de France, volume 102 (2), juin 1997. pp. 93-102; https://www.persee.fr/doc/bsef_0037-928x_1997_num_102_2_17314

\section{Ressources associées :}

Ptilodactyla exotica

Coleoptera

Ptilodactylidae

Fichier pdf généré le 24/09/2019 


\title{
Résumé
}

Résumé. -Une pullulation de Ptilodactyla exotica a été observée pendant trois ans dans un appartement des environs de Lyon. Cette espèce ou des espèces voisines ont déjà été signalées dans des locaux d'habitation ou des serres à Dijon et dans différents pays d'Europe (Allemagne, Slovaquie, Suisse et Italie), ainsi qu'aux USA. L'acclimatation de P. exotica en Europe dans des bâtiments chauffés où elle colonise les plantations est un fait établi. L'espèce est probablement originaire des îles Mascareignes car elle a été trouvée à l'île Maurice et tout récemment à l'île de la Réunion. L'adulte et la larve sont décrits ; des informations sont données sur cette espèce, ce genre et cette famille de coléoptères qui font partie intégrante de la faune de la France métropolitaine et de la Réunion. Par ailleurs, le genre Chaetodactyla est cité pour la première fois de la Guadeloupe.

\begin{abstract}
Summary. -Acclimatization in France of the anthropophilous beetle Ptilodactyla exotica (Coleoptera, Ptilodactylidae). A pullulation of Ptilodactyla exotica was observed three years running in a flat near Lyon. This and similar species have already been found in housing and hothouses in Dijon, as well as in various other European countries (Germany, Slovakia, Switzerland and Italy) and the USA. It is an established fact that $\mathrm{P}$. exotica has become acclimatized in heated buildings in Europe where it colonizes plants. The species is probably native to the Mascarene Islands and it has been found in Mauritius and more recently in Reunion. There is a description of the adult and larva. Information is provided on this species, genus and family of beetle which is an integral part of fauna of Metropolitan France and Reunion. Furthermore, mention is made for the first time of the Chaetodactyla genus in Guadeloupe.
\end{abstract}




\title{
Acclimatation en France de Ptilodactyla exotica, espèce à mœurs anthropophiles (Coleoptera, Ptilodactylidae)
}

\author{
par Henri-Pierre ABERLENC * et Roland AlLEMAND ** \\ * CIRAD-CA, laboratoire de Faunistique et de Taxonomie, UREA, bât. 2, 2477 av. du Val de Montferrand, \\ F - 34032 Montpellier cedex 1 ; e-mail : aberlenc@cirad.fr. ** Université Claude Bernard, C.NRS, \\ laboratoire de Génétique des Populations, 43 bd du 11 Novembre 1918, F - 69622 Villeurbanne cedex
}

Résumé. - Une pullulation de Ptilodactyla exotica a été observée pendant trois ans dans un appartement des environs de Lyon. Cette espèce ou des espèces voisines ont déjà été signalées dans des locaux d'habitation ou des serres à Dijon et dans différents pays d'Europe (Allemagne, Slovaquie, Suisse et Italie), ainsi qu'aux USA. L'acclimatation de $P$. exotica en Europe dans des bâtiments chauffés où elle colonise les plantations est un fait établi. L'espèce est probablement originaire des îles Mascareignes car elle a été trouvée à l'île Maurice et tout récemment à l'île de la Réunion. L'adulte et la larve sont décrits; des informations sont données sur cette espèce, ce genre et cette famille de coléoptères qui font partie intégrante de la faune de la France métropolitaine et de la Réunion. Par ailleurs, le genre Chaetodactyla est cité pour la première fois de la Guadeloupe.

Summary. - Acclimatization in France of the anthropophilous beetle Ptilodactyla exotica (Coleoptera, Ptilodactylidae). A pullulation of Ptilodactyla exotica was observed three years running in a flat near Lyon. This and similar species have already been found in housing and hothouses in Dijon, as well as in various other European countries (Germany, Slovakia, Switzerland and Italy) and the USA. It is an established fact that $P$. exotica has become acclimatized in heated buildings in Europe where it colonizes plants. The species is probably native to the Mascarene Islands and it has been found in Mauritius and more recently in Reunion. There is a description of the adult and larva. Information is provided on this species, genus and family of beetle which is an integral part of fauna of Metropolitan France and Reunion. Furthermore, mention is made for the first time of the Chaetodactyla genus in Guadeloupe.

Mots clés. - Coleoptera, Ptilodactylidae, Ptilodactyla, Chaetodactyla, morphologie larvaire, phénologie, distribution géographique, France, espèce anthropophile, acclimatation, serres.

Les importations fortuites d'insectes exotiques sont relativement fréquentes mais sont rarement suivies d'une implantation, c'est-à-dire que les espèces ne trouvent pas habituellement les conditions d'habitats nécessaires pour s'acclimater. Les activités humaines favorisent cependant l'acclimatation d'insectes, notamment celle d'espèces anthropophiles et tout particulièrement celles qui consomment des denrées. Leur réussite est parfois spectaculaire et ces espèces peuvent devenir de réels fléaux économiques (LEPESME, 1944, DELOBEL \& TRAN, 1993), ce qui n'est heureusement pas le cas avec l'espèce qui nous préoccupe.

En effet, nous avons récemment découvert dans la région lyonnaise un coléoptère Ptilodactylidae : Ptilodactyla exotica Chapin, 1927. Il pullulait dans un appartement où il était capable de se reproduire.

En outre, plusieurs exemplaires de cette même espèce provenant de la Réunion nous ont été communiqués. Ceci nous conduit à attirer l'attention sur cette famille tropicale méconnue des entomologistes français et à dresser le bilan des données dont nous disposons.

\section{Observations}

Des exemplaires adultes de Ptilodactylidae ont été communiqués à l'un d'entre nous (R.A.) à la suite d'une pullulation répétée dans un appartement de la banlieue lyonnaise. Ce logement est situé au troisième étage d'un immeuble récent de Vaulx-en-Velin (Rhône) et ses 


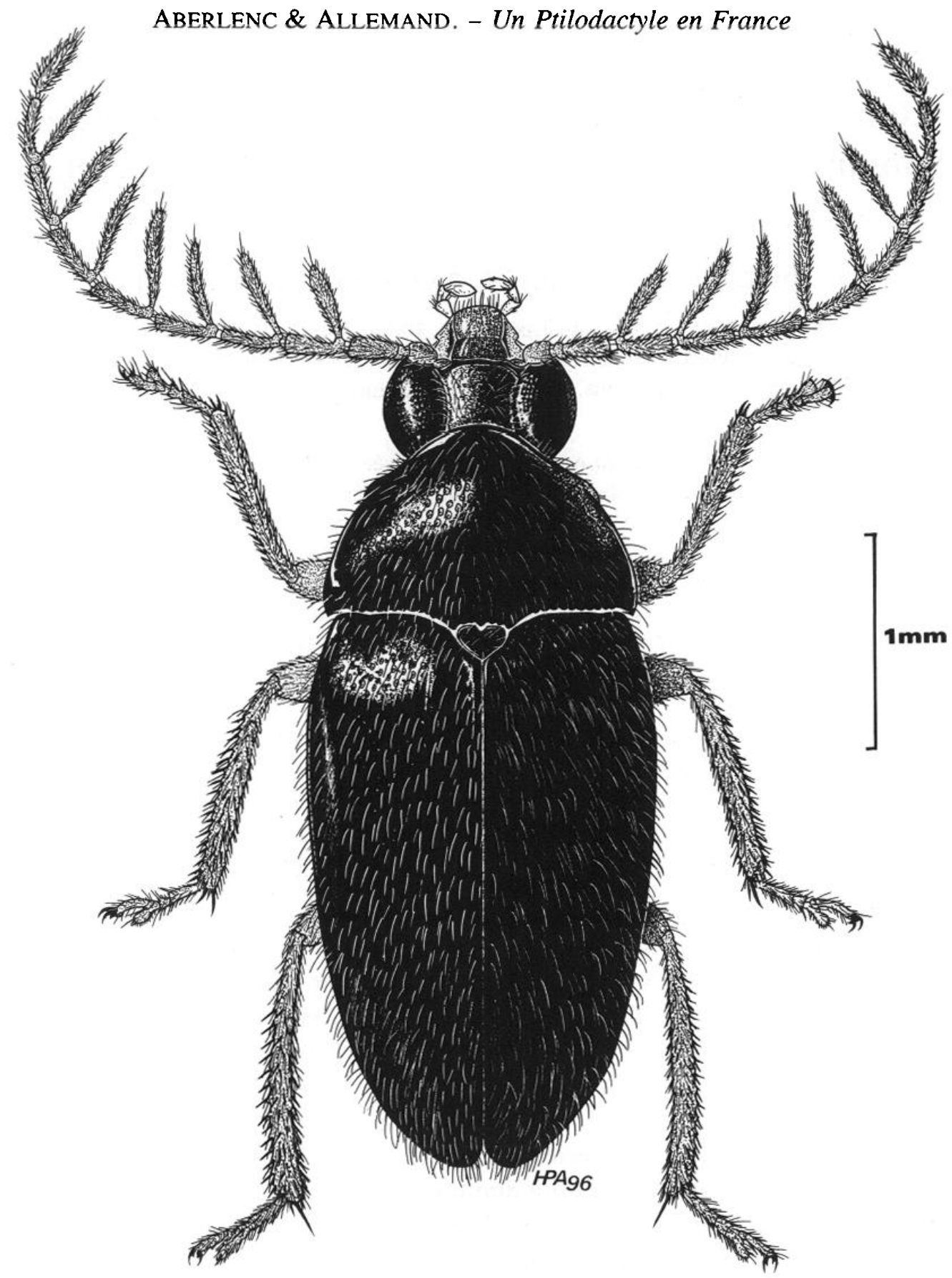

Fig. 1. - Ptilodactyla exotica, ờ (la tête, normalement cachée sous le pronotum, est ici très fortement relevée).

occupants ont observé régulièrement ces insectes pendant trois années, de 1993 à 1995, avant de les éliminer volontairement et définitivement.

Les insectes étaient présents dans la pièce de séjour, orientée au sud, surtout pendant l'été, avec des émergences d'individus pouvant atteindre plusieurs dizaines par jour. En hiver, cet appartement était bien chauffé (chauffage central collectif) et quelques adultes pouvaient être encore aperçus. Ces insectes, essentiellement nocturnes, étaient surtout trouvés près ou sous des bacs de plantes vertes d'appartement. Agiles et capables de voler le soir autour des plantes, ils ne semblaient pas être particulièrement attirés par les fenêtres ni par l'éclairage de la pièce.

Les bacs de plantes, d'un volume d'environ trente litres, possédaient une réserve d'eau et contenaient des espèces banales d'appartement comme Chlorophytum, Codiaeum, Fatsia, Ficus, Monstera et Sansevieria. En janvier 1995, une recherche dans les bacs a révélé la présence de nombreuses larves se développant dans les zones où le terreau était très humide. 
D'après les personnes occupant l'appartement, les insectes sont apparus quelques semaines après le renouvellement du terreau des bacs en mai 1993. Ce terreau ayant été acheté dans un supermarché de la région, il ne fut malheureusement pas possible de connaître sa provenance. La présence des larves et des adultes n'a pas eu d'effets notables sur le développement des plantes.

Par ailleurs, notre collègue $M$. Attié a collecté à la Réunion trois exemplaires de la même espèce que celle de Vaulx-en-Velin (identité spécifique confirmée par examen de l'édéage): 10, Saint-Philippe GR 2 (réf. 334), 21.VIII.1995, sur Bertiera borbonica A. Rich, «espèce arbustive endémique de la Réunion»; 1 \%, Saint-Denis, lieu-dit la Providence (réf. 395), 4.IV. 1996, dans un jardin «en rempotant des plantes avec de la terre provenant d'un terrassement»; 1 \% , idem, 29.VI.1996, dans une maison.

\section{Autres captures de Ptilodactyla}

BERTRAND (1972) signale que la larve de Ptilodactyla luteipennis Pic' «a été trouvée en Europe sur un bananier» sans autre précision. Plus intéressant encore, il écrit que J. Bitsch a obtenu en 1965 à Dijon de nombreux Ptilodactyla d'un pot de Philodendron. Cette remarquable découverte d'un coléoptère appartenant à une famille et un genre nouveau pour la Faune de France a été presque totalement méconnue pendant trente ans! J. Bitsch, que nous avons interrogé, n'a conservé aucune trace écrite ni aucun spécimen de ses captures. Il avait observé des adultes dans un appartement et avait conclu que «ces coléoptères provenaient très probablement du terreau, acheté chez un fleuriste, mis dans un grand pot de terre où poussaient des Philodendron». Bien que ce soit à jamais impossible à prouver, nous pouvons raisonnablement supposer qu'il s'agissait de $P$. exotica.

La rédaction de la diagnose, des données géographiques et biologiques tout comme l'illustration du présent article étaient déjà réalisées quand nous avons pris connaissance de la remarquable synthèse de nos collègues SÜSS \& PUPPIN (1975).

Ils signalent la présence de Ptilodactyla exotica en Italie. L'espèce a été découverte en Lombardic, cn scrrc, sur la Bromćliacćc Aechmea fasciata. Un mâle fut collecté dans une serre où était pratiquée la monoculture de Dracaena fragrans. Le professeur L. Süss nous a aimablement communiqué des échantillons pris à Milan au printemps 1975. Le Ptilodactyla que BINAGHI (1972) signale de la province de Gênes, à Carasco, acclimaté dans des serres chaudes où était pratiquée la monoculture d'Anthurium andreanum, appartient selon les mêmes auteurs à la même espèce. Les premières captures connues dans cette région, à Bogliasco (en serres, sur Anthurium) remontent aux années 1940 !

Le type de CHAPIN (1927) provenait d'une serre de rosiers aux USA, dans l'Illinois, à Melrose Park près de Chicago. D'autres spécimens avaient été trouvés à Washington D.C. et à Willow Grove, en Pennsylvanie, toujours en serre de roses. Le nom que Chapin a choisi souligne qu'il pensait que l'espèce était importée.

SÜSS \& PUPPIN (1975) ont découvert dans la collection Pic, au Muséum national d'Histoire naturelle (Paris), cinq exemplaires indéterminés collectés à l'île Maurice qu'ils ont identifiés : ce sont des P. exotica $(1 q$, Port-Louis, IV.1932; 1 \% , 1 \% , Rose-Hill, XII.1934; 19 , idem, I.1935; 10 , Corps-de-Garde, 10.XII.1932).

Ces deux auteurs en déduisent que l'espèce est originaire de cette île. Nous sommes d'accord avec leur raisonnement. La découverte de $P$. exotica à la Réunion élargit cette aire originelle aux Mascareignes. Il est remarquable que ce soit la seule région connue où l'on rencontre cette espèce dans la nature. Il faut cependant souligner que nous avons affaire à une hypothèse vraisemblable et non à une certitude absolue.

D'après SCHILlHAMMER (1993), un mâle, appartenant sans doute encore à la même

1 D'après Süss \& PUPPIN (1975), il s'agit de $P$. luteipes Pic, «luteipennis» n'étant qu'un lapsus calami de Bertrand. 
espèce (d'après la photo publiée), a été capturé à Bratislava en Slovaquie (8.VI.1988) volant contre une fenêtre; sept autres exemplaires ont été trouvés en Allemagne à l'Université de Bielefeld (Westphalie) dans du bois tropical importé. Cet auteur conclut que l'espèce se rencontrera assez fréquemment dans des serres en Europe. D'après SÜss \& PUPPIN (1975), HORION (1955) avait déjà signalé la présence de Ptilodactyla luteipes Pic en Allemagne dans des serres.

C. Besuchet nous a communiqué des $P$. exotica collectés à Berne en Suisse, qui avaient émergé d'un pot de bambous.

\section{Les Ptilodactylidae}

Cette famille méconnue mérite d'être présentée. Les éléments retenus sont la synthèse des donnćcs bibliographiques (CHAPIN, 1927, SÜSS \& PUPPIN,1975, PARKER, 1982, STRIBLING, 1986 a et b, LAWRENCE \& BRITTON, 1991) et de nos propres observations (matériel provenant de la région lyonnaise, de la Réunion, de Suisse, d'Italie, de Guadeloupe et de Guyane française).

Les Ptilodactylidae montrent une certaine ressemblance avec les Helodidae et les Dascillidae. Leur position systématique est restée longtemps incertaine avant que la famille ne soit définie et placée parmi les Dryopoidea (CROwSON, 1955).

D'après LAWRENCE \& STRIBLING (1992), cette famille comprend environ 25 genres et 500 espèces répandues dans le monde entier mais principalement en zone tropicale.

Les adultes de Ptilodactylidae ont une taille pouvant varier de 4 à $12 \mathrm{~mm}$, voire de 2 à $16 \mathrm{~mm}$. Leur corps est ovale plus ou moins allongé, parfois subparallèle, convexe, de couleur brun plus ou moins foncé, glabre ou couvert de soies courtes. La tête est généralement non ou peu visible de dessus, enfoncée dans le prothorax, la bouche orientée vers le bas. Les antennes, assez longues, sont filiformes, serrulées ou pectinées, la pectination étant articulée ou non chez le mâle : le dimorphisme sexuel antennaire est la règle chez la plupart des espèces. Le clypéus est transverse et ne cache ni le labre ni les mandibules. Le pronotum est rebordé latéralement, ce rebord atteignant ou non les angles antérieurs. Le bord postérieur du pronotum est soit crénelé, soit lisse. Les cavités coxales antérieures sont ouvertes. Les élytres, recouvrant tout l'abdomen, sont non ou faiblement striés, rarement avec des stries en relief. L'écusson est soit cordiforme, le bord antérieur étant entaillé, soit simplement triangulaire. Le tarse est toujours pentamère (formule tarsale 5-5-5). Dans certains cas, il est simple. Dans d'autres cas, les troisièmes tarsomères seulement, plus rarement les seconds et troisièmes tarsomères ensemble, sont lobés en dessous, d'où les noms latin (Ptilodactylidae signifiant «tarse ailé») et anglo-saxon («toed-winged beetles») de la famille; le quatrième article est alors très petit, si bien que la formule tarsale semble être 4-4-4. Les griffes sont simples, bifides ou pectinées.

Les larves peuvent atteindre 10 à $15 \mathrm{~mm}$ au dernier stade. Elles sont allongées, subcylindriques, parfois déprimées dorsalement et portent quelques soies. La tête, la face dorsale du thorax et parfois des segments abdominaux I à IX sont plus sclérifiées et pigmentées que les autres parties, la sclérification et la pigmentation pouvant même s'étendre sur presque tout le corps. La tête est prognathe et partiellement rétractile dans le prothorax. Elle porte des antennes de trois articles dont le troisième est très petit. Près de la base des antennes sont présentes des ommatidies sur des taches pigmentées. Le labre est bien distinct, les mandibules sont de taille moyenne, généralement tridentées à l'apex au bord interne. La maxille a un palpe tri- ou quadrisegmenté, üne galéa bisegmentée et une lacinia sétifère. Le labium a un mentum bien distinct et des palpes bisegmentés. La suture gulaire est bien visible. En comptant la coxa et la griffe apicale unique, les pattes ont cinq articles. L'abdomen est formé de dix segments ; le neuvième, souvent en position apicale, est différent des précédents et porte un dixième segment qui n'est généralement pas dans l'axe du corps. Des structures particulières encadrent l'anus. Les stigmates sont biperforés et chez certaines espèces aquatiques des branchies sont présentes sur l'abdomen. 


\section{Description de Ptilodactyla exotica Chapin}

Nos échantillons (mentionnés ci-dessus), appartiennent au genre Ptilodactyla Illiger, (M.A. Jäch det., confirmation par la clé de STRIBLING, 1986a), sous-famille des Ptilodactylinae. La détermination spécifique est faite d'après SüsS \& PUPPIN (1975), qui ont décrit l'imago et la larve, puis confirmée par l'examen de leurs propres échantillons. D'après STRIBLING (1986a), ce genre compte 270 espèces dans le Nouveau Monde. PIC (1914) recensait par ailleurs une espèce en Nouvelle-Calédonie et à Vanuatu, une à Java, une à Ceylan et une au Japon.

$L$ 'imago est de couleur brun foncé presque noire avec la face ventrale moins sombre, les antennes brunes et les pattes plus claires (fig. 1). La longueur varie de 3,5 à $6 \mathrm{~mm}$ (tête non comprise). Les quatre exemplaires de Milan que nous avons examinés sont plus pâles, plus testacés.

Dimorphisme sexuel : les femelles sont plus trapues et plus grandes que les mâles; les yeux sont gros et subsphériques, plus gros et plus rapprochés chez les mâles; les antennes des mâles portent de remarquables expansions latérales articulées à la base des segments 4 à 10 (fig. 1), tandis que celles des femelles sont serrulées (fig. 2). Les griffes sont bifides, avec une dent basale qui est plus forte et plus pointue chez le mâle (fig. 5).

Le tégument est lisse et brillant sur la face dorsale avec une pubescence jaune, assez dense, couchée et relativement courte. Les soies sont insérées sur des reliefs circulaires, creusés au centre.

La tête est presque entièrement cachée en vue dorsale car elle est insérée sous le pronotum. La suture frontoclypéale est incurvée et forme une carène relevée latéralement derrière l'insertion antennaire. La gula est nettement visible, dessinant la silhouette d'une «coupe à champagne» en arrière du labium.

La mandibule montre, en allant de l'insertion à l'extrémité : la base, très large, qui présente dorsalement une haute carène tranchante subtriangulaire en vue latérale; la mandibule se rétrécit ensuite brusquement, puis s'incurve fortement vers l'intérieur; l'apex est bidenté, mais ce caractère ne peut être observé en vue dorsale car la dent supérieure, dans l'axe, est plus longue que la dent inférieure qui est inclinée vers le bas.

Le pronotum est bombé, moins large et arrondi en avant, formant une "visière" lisse et étroite au-dessus de la têtc. Lcs côtés sont sinués avec une carène tranchante partant de l'angle postérieur et s'effaçant avant le bord antérieur. Les angles postérieurs sont légèrement saillants en arrière et forment un «bec d'aigle». Le bord postérieur est finement crénelé (sauf de part et d'autre de l'écusson), il présente une triple sinuosité concave coaptée aux bords antérieurs convexes des élytres et de l'écusson.

Les stries élytrales sont très effacées et difficiles à voir, elles forment de légers sillons avec des points enfoncés.

Les hanches antérieures sont globuleuses, saillantes, subconiques. Le mésosternum porte une fine carène médiane vers l'arrière, entre les hanches intermédiaires. Les hanches postérieures sont transverses-obliques et concaves pour recevoir les métafémurs. Les pattes sont fortement poilues, les tibias étant armés de courtes épines sur presque toute leur longueur, à l'exception de la base.

Le tarse est pentamère mais semble tétramère: le premier article est long; le second est bien plus court, ces deux premiers articles étant obliquement tronqués à l'apex; le troisième est fortement lobé vers le bas; le quatrième est minuscule, inséré sur le dessus et près de la base du troisième ; le cinquième article est allongé (fig. 5).

L'abdomen a cinq sternites visibles, le premier étant fortement découpé au milieu par les hanches postérieures et le cinquième étant très légèrement échancré à l'apex dans les deux sexes, notre appréciation s'écartant sur ce point de celles de CHAPIN (1927) et de Süss \& PUPPIN (1975).

Edéage: voir figures 3 et 4 .

Les larves du dernier stade sont allongées et subcylindriques, le diamètre de tous les segments étant presque égal (fig. 6). Elles sont de couleur jaunâtre pâle, peu sclérifiées sur les faces ventrale et latérales. La tête, la face dorsale du thorax (en particulier l'avant du pronotum), ainsi que le neuvième segment abdominal sont plus sclérifiés et de couleur rousse. La séparation de la face sternale et de la face tergale est nette. 
Les mandibules sont tridentées et fortement sclérifiées. Elles portent à la base, sur la face interne, un remarquable processus articulé, allongé et minuscule, terminé par un bouquet de soies qui lui donnent l'aspect d'un "pinceau" : la prosthèque (ce caractère n'est visible que par transparence entre lame et lamelle, car il est caché entre le labre et la maxille). Les maxilles sont formées d'un grand cardo subtriangulaire, d'un stipe nettement visible en forme de losange, d'un palpe quadrisegmenté, d'une galéa bisegmentée et d'une lacinia sétifère. Les palpes labiaux sont bisegmentés (fig. 8).

Le prothorax est presque aussi long que les méso et métathorax pris ensemble.

Le neuvième segment abdominal est subconique, bombé dorsalement et incurvé ventralement. Le dixième segment abdominal, situé sous le neuvième, est très peu sclérifié et frangé de soies; il porte deux petites protubérances symétriques de chaque côté de l'anus, terminées chacune par un "peigne" de 6, 7 ou 8 épines que Süss \& PUPPIN (1975) appellent les "formations spiniformes" (fig. 9).

Les stigmates sont situés latéralement sur le mésothorax et les huit premiers segments abdominaux, le mésothoracique étant nettement plus gros que les autres. Il sont tous biperforés (fig. 6, 10).

Le mésothorax et le huitième segment abdominal portent chacun une paire de petites glandes latérales évaginables ${ }^{2}$ situées à côté du stigmate, caractère qui semble être propre aux Ptilodactyla.

Par leur forme cylindrique, leur comportement et leur milieu, ces larves rappellent grossièrement celles de certains Elateridae ou Tenebrionidae.

\section{Biologie des Ptilodactylidae}

La biologie des Ptilodactyles est encore mal connue. On les trouve dans des biotopes humides, voire marécageux ou périodiquement inondés.

Les adultes fréquentent la végétation arbustive. On peut aussi rencontrer certaines espèces sur du bois coupé récemment ou non. Ils peuvent être parfois attirés par la lumière.

Les larves de certaines espèces sont aquatiques et munies de branchies, d'autres vivent dans le bois humide en décomposition, dans la litière ou dans le terreau. Des larves aquatiques de Ptilodactylidae ont été trouvées dans des feuilles immergées en décomposition, à découvert en eaux stagnantes ou courantes, dans la végétation aquatique, sur des bois immergés ou encore «dans le sable, au fond des torrents, rongeant des racines» (BERTRAND, 1972).

Cet auteur écrit aussi que les larves de Ptilodactyla "se rencontrent dans la terre rongeant les racines de divers végétaux particulièrement dans les régions tropicales, si bien que souvent ces larves ont été introduites dans des serres chaudes». Rappelons que, d'après nos observations, les larves de Ptilodactyla ne semblent causer aucun dommage aux plantes des pots où elles vivent. Les larves de Ptilodactyla se développent en milieu humide, dans le terreau, le bois en décomposition et dans la litière, éventuellement aux dépens de racines plus ou moins décomposées. D'après Süss \& PUPPIN (1975), leur présence dans les serres italiennes semble liée à certaines espèces de plantes ou à la nature du substrat.

Certaines espèces de Ptilodactylidae sont xylophages, sur bois décomposé ou tendre.

STRIBLING \& SEYMOUR (1988) ont établi que certains Ptilodactylidae adultes sont au moins en partie mycophages (genres Aploglossa, Ptilodactyla, Lachnodactyla. Stirophora et Daemon). Leur lacinia est transformée en une brosse à spores. L'examen du contenu de leur intestin moyen et postérieur a révélé que la majorité des spores et des hyphes d'Ascomycètes détectés étaient pigmentés. Cela démontre une ingestion volontaire et préférentielle de ces espèces à spores sombres, car leur substrat contient de nombreuses espèces fongiques à spores claires. Les espèces choisies produisent toutes des stuctures assez grandes, ce qui les rend peut-être plus faciles à découvrir... et à consommer par ces coléoptères.

2 Rien ne nous permet de savoir à l'heure actuelle si ces glandes ont une fonction défensive ou si elles permettent une communication chimique entre larves. Elles font penser, mutatis mutandi, aux glandes exsertiles de certains coléoptères Malachiidae. 


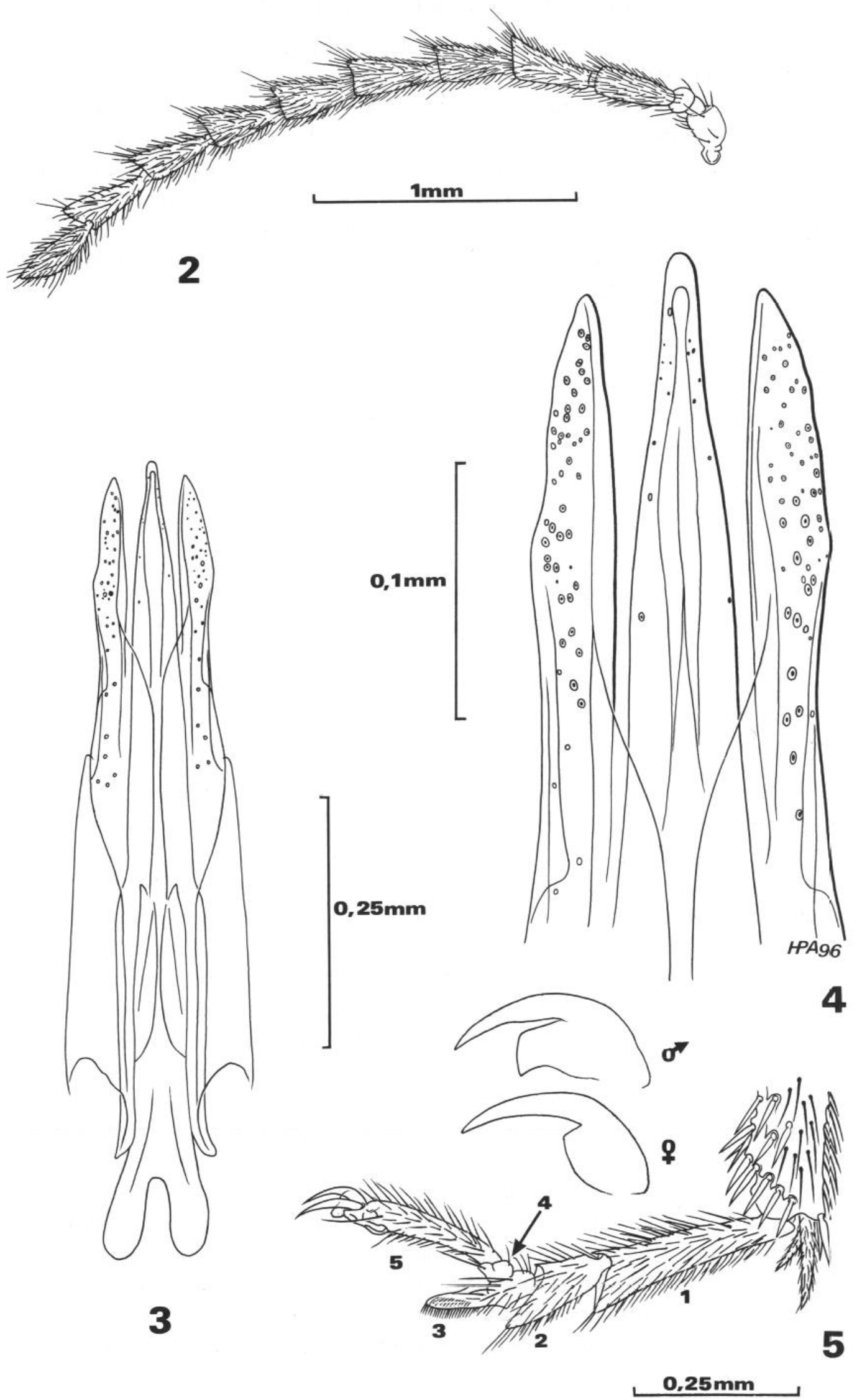

Fig. 2-5. - Ptilodactyla exotica, morphologie imaginale. - 2, antenne de la femelle ; - 3, édéage ; - 4, apex de l'édéage : -5 . tarse intermédiaire de la femelle et griffe du tarse antérieur du mâle et de la femelle. 


\section{Observations complémentaires de Ptilodactylidae en Région Néotropicale}

Nos déterminations s'appuient sur la clé des genres de Ptilodactylidae du Nouveau Monde de STRIBLING (1986a).

En Guyane française à Petit-Saut en octobre 1989, trois espèces de Ptilodactyla ont été collectées par l'un d'entre nous (H.-P.A.) : un mâle le 20 octobre, trouvé sous les écorces d'un arbre abattu et décomposé dans un secteur où la forêt primaire avait été coupée ; un mâle pris au piège Malaise (24 au 31 octobre) en sous-bois de forêt primaire, dans un petit chablis naturel ; enfin une femelle découverte le 30 octobre en forêt primaire.

Nous avons également collecté cinq larves de Ptilodactylidae : le 28 octobre, une larve de $11 \mathrm{~mm}$ dont le neuvième segment porte une paire de forts urogomphes, sous les écorces d'un arbre mort; deux larvcs d'unc cspc̀cc dont lc neuvième segment abdominal est mucroné (une jeune de $4 \mathrm{~mm}$ et une plus âgée de $8,5 \mathrm{~mm}$ ); le 23 octobre, dans le terreau accumulé sous les écorces déhiscentes d'un arbre abattu au-dessus d'une «crique» (petit ruisseau) en sous-bois de forêt primaire, une larve de $6 \mathrm{~mm}$ au neuvième segment mucroné qui appartient probablement au même genre mais sans doute pas à la même espèce que les deux précédentes et une larve de Ptilodactyla $s p$. Les quatre premières ont le stigmate mésothoracique en position ventrale.

La faune des Ptilodactylidae de Guyane doit être riche, car à l'époque nous ne les cherchions pas spécialement !

En Guadeloupe, à Capesterre-Neufchâteau, notre collègue $J .-M$. Maldès a pris le 6 juin 1996 deux mâles d'un Chaetodactyla qui est vraisemblablement $C$. lyciformis Champion, 1897 à l'aide d'un piège d'interception (DEGALLIER \& ARNAUD, 1995), en lisière de la forêt, à côté d'une bananeraie. Ces captures sont très intéressantes, car la répartition de l'unique espèce connue de ce genre s'étend d'après STRIBLING (1986a) du Nicaragua à la Colombie : c'est la première citation des Antilles. Seul l'examen de l'édéage permettrait d'être certain de l'identité spécifique de nos Chaetodactyla, mais nous ne disposons pas de matériel de comparaison provenant du continent.

\section{Conclusion}

Le développement et la reproduction de $P$. exotica nécessite des matières végétales décomposées et humides placées dans une atmosphère chaude, peut-être aussi la présence de certains Ascomycètes. Ces conditions sont remplies dans les serres chaudes mais également dans les habitations où les bacs à plantes à réserve d'eau constituent un milieu très favorable. Compte tenu de cette biologie, la dissémination par le terreau ou par des rejets de plantes repiqués avec un peu de sol d'origine semble être assez facile et cette espèce est peut-être déjà relativement répandue. En absence de pullulation, l'espèce peut facilement passer inaperçue en raison de ses mœurs plutôt nocturnes et par sa ressemblance superficielle, par la taille et la coloration, avec des spécimens d'Attagenus (Dermestidae), souvent présents dans les habitations. Dans le cas de Vaulx-en-Velin, si l'hypothèse de la contamination par du terreau provenant d'un supermarché est exacte, l'espèce a dû être disséminée dans de nombreux sites. Elle semble ne pas être très gênante et n'a aucune importance économique.

Il est certain que $P$. exotica, vraisemblablement originaire des Mascareignes, est régulièrement importé en Europe et aux USA où il peut s'installer et se maintenir pendant plusieurs générations et plusieurs années. C'est une acquisition très intéressante pour la Faune de la France et de la Réunion. Nous ne pouvons qu'encourager nos collègues à le rechercher sous nos latitudes et, au cours de leurs prospections sous les tropiques, à s'intéresser à cette attachante famille méconnue. 

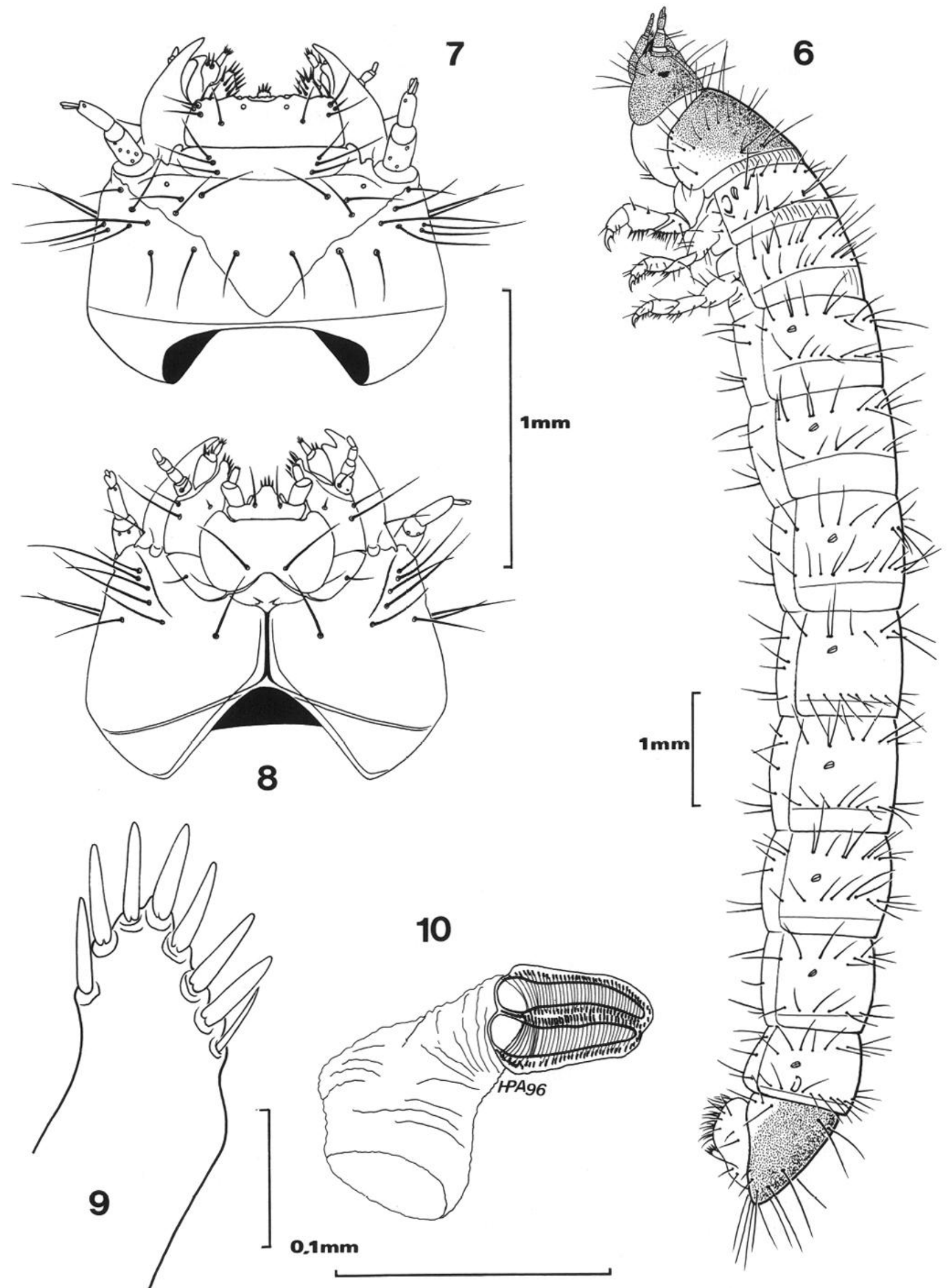

Fig. 6-10. - Ptilodactyla exotica, morphologie du dernier stade larvaire. - 6, habitus; - 7, tête (vue dorsale); -8 , tête (vue ventrale); - 9, apex du «peigne" du dixième segment abdominal; - 10, stigmate mésothoracique. 
REMERCIEMENTS. - Nous adressons nos remerciements à Mlle S. Janillon qui nous a soumis les premiers exemplaires de cet insecte, à $\mathrm{M}$. et Mme J. Martinez qui nous ont fourni les renseignements et permis les observations à Vaulx-en-Velin, ainsi qu'à $\mathbf{M}$. Matthew Daillie pour sa traduction. Nous remercions également nos collègues $M$. Attié de l'Université de la Réunion, $C$. Besuchet du Muséum de Genève, J. Bitsch de l'Université Paul Sabatier de Toulouse, G. Curletti du Museo Civico di Storia Naturale de Carmagnola, A. Jäch du Muséum de Vienne, R. Roy du Muséum de Paris et L. Süss de l'Istituto di Entomologia Agraria de Milan.

\section{AUTEURS CITÉS}

BERTRAND H., 1972. - Larves et nymphes des coléoptères aquatiques du globe. Paris: édité par l'auteur, 804 pp.

BiNAGHI G., 1972. - Sulla presenza di un Coleottero Ptilodactylidae nelle coltivazioni di Anthurium da fiore in serre calda nella provincia di Genova. Bollettino della Societa entomologica italiana, $104(1-3)$ : 40-43.

Chapin E. A., 1927. - The North American species of Ptilodactyla (Coleoptera: Helodidae). Transactions of the American entomological Society, 53 : 241-249.

Crowson R. A., 1955. - The natural classification of the families of Coleoptera. Hampton: Classey, réédition 1967, $214 \mathrm{pp}$.

Degallier N. \& ARNAud P., 1995. - Utilisation du piège d'interception. Bulletin de l'ACOREP, $23: 57-58,1$ fig.

Delobel A. \& TRAN M., 1993. - Les Coléoptères des denrées alimentaires entreposées dans les régions chaudes. Paris: ORSTOM et CTA, 425 pp., 145 fig.

HoRION A., 1955. - Faunistik der Mitteleuropaïschen Käfer. IV. Sternoxia, Fossipedes, macrodactyla, Brachymera. München : Reitter, cf. p. 138.

LAWRENCE J.F. \& Britron E.B., 1991. - Coleoptera. In CSIRO, The insects of Australia, 2nd. Edition, Melbourne : Melbourne University Press, $1137 \mathrm{pp}$.

LAWRENCE J.F. \& STRIBling J.B., 1992. - A new genus of Ptilodactylidae (Coleoptera: Elateriformia) from North queensland, with description of the presumed larva. Journal of the Australian Entomological Society, 31 (1) : 19-27, 19 fig.

LEPESME P., 1944. - Les Coléoptères des denrées alimentaires et des produits industriels entreposés. Paris : Lechevalier, 335 pp., 232 fig., 11 pl.

PARKER S. P. ed., 1982. - Synopsis and Classification of Living Organisms. Part 2. New York: Mc Graw Hill Book Company, 1232 pp., 141 pl.

PIC M., 1914. - Dascillidae, Helodidae, Eucinetidae. In Coleopterorum Catalogus, X, pars 58. Berlin : Junk, $65 \mathrm{pp}$.

SCHILlHAMMER H., 1993. - Bemerkenswerte Käferfunde aus Österreich (II) (Coleoptera). Koleopterologische Rundschau, 63 : 325-332, 3 fig.

STRIBling J.B., 1986a. - Revision of Anchytarsus (Coleoptera: Dryopoidea) and a key to the New World genera of Ptilodactylidae. Annals of the Entomological Society of America, 79 (1) : 219234,48 fig.

- 1986b. - World generic revision of Ptilodactylidae (Coleoptera: Dryopoidea). Ph.D. thesis, Ohio State University.

StRIBLING J.B. \& SEYMOUR R.L., 1988. - Evidence of mycophagy in Ptilodactylida (Coleoptera : Dryopoidea) with notes on phylogenetic implications. The Coleopterists Bulletin, 42 (2) : 152-154.

Süss L. \& PuPPIN O., 1975-76. - Osservationi sulla morfologia e sulla biologia de Ptilodactyla exotica Chapin (Coleoptera, Ptilodactylidae) nelle serre della Lombardia e contributo bibliographico allo studio del grupo. Bollettino di Zoologia agraria e Bachicoltura, Ser. II, 13 : $143-165,14$ fig. 